Rafael Corzo de la Colina ${ }^{(*)}$ y José Villafuerte Mendoza ${ }^{(* *)}{ }^{(* *)}$

\title{
Seguros de grandes riesgos y protección desproporcionada de los asegurados en la Ley del contrato de seguros ("m)
}

\section{Great risk insurances and disproportionate protection of insured persons in insurance contract Law}

\author{
LA ASIMETRÍA INFORMATIVA EN UN MERCADO SIGNIFICA, A GRANDES RASGOS, QUE LOS \\ CONSUMIDORES CONOCEN O CUENTAN CON MENOS INFORMACIÓN QUE LOS PROVEEDORES \\ SOBRE UN DETERMINADO PRODUCTO O SERVICIO. DENTRO DEL MERCADO DE SEGUROS, \\ LA ASIMETRÍA INFORMATIVA SE VE REFLEJADA PRINCIPALMENTE EN LA COMPLEJIDAD Y \\ ESPECIALIZACIÓN DE LOS CONTRATOS QUE LIMITARÍAN LA POSIBILIDAD DEL CONSUMIDOR \\ PARA PODER TOMAR UNA DECISIÓN DE CONSUMO DEBIDAMENTE INFORMADA.
}

\begin{abstract}
Resumen: En el presente artículo, los autores nos hablan de los seguros de grandes riesgos y describen su tratamiento en la legislación extranjera. Luego, señalan que el rol protector del Estado en la relación de consumo es reducir las asimetrías informativas, pero no existe una obligación total de divulgación de información en el mercado. Por lo tanto, la asimetría informativa deja de ser relevante cuando el usuario del servicio tenga capacidad de negociación y recursos suficientes para tomar una decisión informada. Concluyen que es pertinente equiparar la aplicación de la Ley del Contrato de Seguro peruana a estándares internacionales.
\end{abstract}

Palabras clave: Ley del Contrato De Seguros - Seguro de Grandes Riesgos - Rol Protector del Estado - Asimetría Informativa - Proveedores y Consumidores - Sobrerregulación

Abstract: In this article, the authors tell us about great risk insurances and describe its regulation in foreign law. Then, they point out the protective role

(*) Abogado por la Universidad Católica Santa María. Socio del Área de Seguros y Reaseguros de Miranda \& Amado Abogados Correo electrónico: rcorzo@mafirma.com.pe

$\left.{ }^{* *}\right)$ Abogado por la Universidad Católica de Santa María. Asociado del Área de Seguros y Reaseguros de Miranda \& Amado Abogados. Correo electrónico: jvillafuerte@mafirma.com.pe

$\left.{ }^{(* *}\right)$ Los autores agradecen al doctor Eduardo Wiese Bazo, Consejero del Área de Seguros y Reaseguros de Miranda \& Amado Abogados, por los valiosos aportes y sugerencias hechas a este artículo.

${ }^{* * * *}$ Nota del Editor: este artículo fue recibido el 15 de junio del 2017 y su publicación fue aprobada el 28 de julio del mismo año. 


\section{Seguros de grandes riesgos y protección desproporcionada de los asegurados en la Ley del contrato de seguros Great risk insurances and disproportionate protection of insured persons in insurance contract Law}

of State in the consumption relationship is to reduce information asymmetries, but there is no total disclosure obligation in the market. Therefore, information asymmetry ceases to be relevant when the user of the service has negotiating capacity and necessary resources to make an informed decision. They conclude it is pertinent to equate the application of the Peruvian Insurance Contract Law to international standards.

Keywords: Insurance Contract Law - Great Risk Insurance Protective Rol of State - Information Asymmetry - Providers and Consumers - Over-Regulation

El 22 de junio de 2015 fue presentado ante el Congreso de la República el Proyecto de Ley 4635/2014-CR, el mismo que proponía modificar el Artículo I del Título I (Disposiciones Generales) de la Ley 29946, Ley del Contrato de Seguro (la "Ley del Contrato de Seguro"), incorporando el siguiente párrafo:

"Exceptúese de lo anterior [la aplicación de la Ley del Contrato de Seguro] a los seguros de grandes riesgos. Se consideran seguros de grandes riesgos a los seguros patrimoniales contratados por personas jurídicas que cumplan con las características establecidas por la Superintendencia de Banca, Seguros y AFP" (la cursiva es agregada).

El referido Proyecto de Ley no tuvo dictamen alguno en las Comisiones del Congreso de la República y concluida la legislatura (2011-2016) fue remitido al archivo. Este proyecto tuvo cierta repercusión mediática en aquel momento y generó un debate sobre la necesidad y legitimidad de excluir a los seguros que cubran a los grandes riesgos del ámbito de protección y los alcances de la Ley del Contrato de Seguro.

En el presente artículo analizaremos el concepto y las características principales de los grandes riesgos y revisaremos su tratamiento legislativo, con la finalidad de poder determinar si su exclusión del ámbito de protección y de los alcances de la Ley del Contrato de Seguro es necesaria y legítima.

\section{Definición de Grandes Riesgos}

Como aspecto preliminar, debemos tener en cuenta que la definición de seguros de grandes riesgos no tiene una connotación unívoca entre la doctrina o la legislación comparada, y que el sentido de dicho término dependerá del tratamiento legislativo que adopte cada país con relación a este tipo de seguros. Sin embargo, de la revisión de diversas legislaciones (España, Francia y Chile), podemos extraer cuatro características comunes entre las distintas definiciones de grandes riesgos:

a) Se tratan de riesgos cuyo valor es superior al promedio y que, normalmente, no se encuentran relacionados con el mercado retail.

b) Son riesgos cuya determinación se encuentra sujeta a criterios objetivos establecidos en la legislación (tipos de riesgos, valor de la prima, monto cubierto por el seguro, condiciones económicas y financieras del asegurado, entre otros aspectos y criterios).

c) Se tratan de riesgos cuya cobertura y regulación no se encuentran sometidos a la aplicación de las normas sobre protección del consumidor, ni a normas especiales sobre protección a favor de los tomadores de seguros.

d) La relación entre asegurado y asegurador se encuentra limitada únicamente por los términos de sus contratos, que han sido redactados y negociados en ejercicio de la autonomía de la voluntad de las partes y su libertad contractual.

\section{Tratamiento legislativo comparado}

\subsection{España}

El artículo 2 de la Ley del Contrato de Seguro española, Ley 50/1980, prescribe que "las distintas modalidades del contrato de seguro, en defecto de Ley que les sea aplicable, se regirán por la presente Ley, cuyos preceptos 


\section{Rafael Corzo de la Colina y José Villafuerte Mendoza}

tienen carácter imperativo, a no ser que en ellos se disponga otra cosa. No obstante, se entenderán válidas las cláusulas contractuales que sean más beneficiosas para el asegurado".

En ese sentido, la misma norma establece en su artículo 44 que "no será de aplicación a los contratos de seguros por grandes riesgos, tal como se delimitan en esta Ley, el mandato contenido en el artículo 2 de la misma".

Al respecto, la Ley 20/2015 establece la definición de grandes riesgos de la siguiente manera:

\section{"Artículo 11. Grandes riesgos}

A efectos de lo establecido en esta Ley y en las demás disposiciones reguladoras de la supervisión y contratación de los seguros privados, se entenderá por contratos de seguro de grandes riesgos los siguientes:

a) Los de vehículos ferroviarios, vehículos aéreos, vehículos marítimos, lacustres y fluviales, mercancías transportadas (comprendidos los equipajes y demás bienes transportados), la responsabilidad civil en vehículos aéreos (comprendida la responsabilidad del transportista) y la responsabilidad civil de vehículos marítimos, lacustres y fluviales (comprendida la responsabilidad civil del transportista).

b) Los de crédito y de caución, cuando el tomador y el asegurado ejerzan a título profesional una actividad industrial, comercial o liberal y el riesgo se refiera a dicha actividad.

c) Los de vehículos terrestres (no ferroviarios), incendio y elementos naturales, otros daños a los bienes, responsabilidad civil en vehículos terrestres automóviles (comprendida la responsabilidad del transportista), responsabilidad civil en general y pérdidas pecuniarias diversas, siempre que el tomador supere los límites de, al menos, dos de los tres criterios siguientes:

- Activo total del balance: 6.200 .000 euros.

- Importe neto del volumen de negocios: 12.800 .000 euros. - Número medio de empleados durante el ejercicio: 250 empleados.
Si el tomador del seguro formara parte de un grupo de sociedades cuyas cuentas consolidadas se establezcan con arreglo a lo dispuesto en los artículos 42 a 49 del Código de Comercio, los criterios mencionados anteriormente se aplicarán sobre la base de las cuentas consolidadas" (la cursiva es agregada).

Como se puede apreciar, la definición de grandes riesgos para la legislación española se encuentra delimitada por tres diferentes condiciones: (i) la naturaleza del riesgo; (ii) el tipo de actividad del tomador; y, (iii) las condiciones económicas y financieras del tomador.

\subsection{Chile}

En Chile, por su parte, la Ley 20667, publicada el 9 de mayo de 2013, que regula el Contrato de Seguro y reemplaza el Título VIII del Libro II del Código de Comercio, establece lo siguiente:

"Art. 542. Carácter imperativo de las normas

Las disposiciones que rigen al contrato de seguro son de carácter imperativo, a no ser que en éstas se disponga otra cosa. No obstante, se entenderán válidas las estipulaciones contractuales que sean más beneficiosas para el asegurado o el beneficiario.

Exceptúense de lo anterior, los seguros de daños contratados individualmente, en que tanto el asegurado como el beneficiario, sean personas jurídicas y el monto de la prima anual que se convenga sea superior a 200 unidades de fomento ${ }^{(1)}$, y los seguros de casco y transporte marítimo y aéreo" (la cursiva es agregada).

En ese sentido, la legislación chilena habría optado por una regulación de grandes riesgos 


\section{Seguros de grandes riesgos y protección desproporcionada de los asegurados en la Ley del contrato de seguros Great risk insurances and disproportionate protection of insured persons in insurance contract Law}

determinada principalmente por el valor de la prima anual convenida, así como en el caso de ciertos riesgos especializados, como sería el caso, por ejemplo, de los seguros de casco y los seguros de transporte marítimo y aéreo.

\subsection{Legislación Comunitaria Europea}

De conformidad con la Directiva 2009/138/CE dictada por el Parlamento Europeo y del Consejo, de fecha 25 de noviembre de 2009, sobre el acceso a la actividad de seguros y de reaseguros y su ejercicio (Solvencia II), se han definido a los grandes riesgos como:

a) Los riesgos clasificados en los ramos de vehículos ferroviarios, vehículos aéreos, marítimos, lacustres y fluviales, mercancías transportadas, responsabilidad civil en vehículos aéreos y responsabilidad civil de vehículos marítimos, lacustres y fluviales.

b) Los riesgos clasificados en los ramos de Crédito y de Caución, cuando el tomador ejerza, a título profesional, una actividad industrial, comercial o liberal, y el riesgo se refiera a dicha actividad.

c) Los riesgos clasificados en los ramos de vehículos terrestres (no ferroviarios), incendio y elementos naturales, otros daños a los bienes, responsabilidad civil en vehículos terrestres automóviles, responsabilidad civil general y pérdidas pecuniarias diversas, siempre que el tomador supere los límites de, al menos, dos de los siguientes criterios:

c.1. Un balance total de 6.200 Millones de Euros en activos. c.2. Un volumen de negocios neto de 12.800 Millones de Euros.

c.3. Un número medio de 250 empleados durante el ejercicio.

Asimismo, si el tomador del seguro forma parte de un grupo de empresas para el que se elaboran cuentas o estados financieros consolidados, los criterios establecidos en el literal c) anterior, se aplicarán sobre la base de las cuentas o estados financieros consolidados.

Al respecto, podemos notar que la legislación española antes descrita ya se encontraría adecuada a la regulación comunitaria, pues compartirían elementos comunes en la determinación y definición de lo que son y el tratamiento de los grandes riesgos para efectos de la industria de los seguros y los reaseguros.

\section{Justificación del rol protector del Estado en el Perú}

El artículo 65 de la Constitución Política vigente dispone que "el Estado defiende el interés de los consumidores y usuarios. Para tal efecto, garantiza el derecho a la información sobre los bienes y servicios que se encuentran a su disposición en el mercado. Asimismo, vela, en particular, por la salud y la seguridad de la población".

A partir de este marco constitucional, el Artículo II del Título Preliminar del Código de Protección y Defensa del Consumidor, aprobado mediante la Ley 29571, establece que:

"El presente Código tiene la finalidad de que los consumidores accedan a productos y servicios idóneos y que gocen de los derechos y los mecanismos efectivos para su protección, reduciendo la asimetría informativa, corrigiendo, previniendo o eliminando las conductas y prácticas que afecten sus legítimos intereses. En el régimen de economía social de mercado establecido por la Constitución, la protección se interpreta en el sentido más favorable al consumidor, de acuerdo a lo establecido en el presente Código" (la cursiva es agregada).

De las normas antes citadas se puede apreciar que el objetivo del rol protector del Estado respecto de los consumidores y los usuarios de productos o servicios sería el de reducir la brecha de asimetría informativa y de corregir las prácticas que puedan afectar sus intereses individuales. 


\section{Rafael Corzo de la Colina y José Villafuerte Mendoza}

Según la definición contenida en el Código de Protección y Defensa del Consumidor, Título Preliminar, Artículo IV, la asimetría informativa es la "característica de la transacción comercial por la cual uno de los agentes, el proveedor, suele tener mayor y mejor información sobre los productos y servicios que ofrece en el mercado a los consumidores".

En la doctrina nacional se ha considerado que la asimetría informativa existe cuando:

"una categoría de agentes económicos -los proveedoresse encuentran en una posición ventajosa frente a la otra -los consumidores o usuarios- como resultado de su capacidad para adquirir y procesar información, consecuencia de su experiencia en el mercado y de su situación frente al proceso productivo. De esta manera, quien conduce un proceso productivo o de comercialización de bienes y servicios en un mercado determinado, cuenta con la posibilidad de adquirir y utilizar de mejor manera la información relevante en dicho mercado, y con ello, eventualmente obtener una ventaja que podría ser utilizada en contravención a la ley" (Espinoza 2004, 150).

En tal sentido, la asimetría informativa en un mercado significa, a grandes rasgos, que los consumidores conocen o cuentan con menos información que los proveedores sobre un determinado producto o servicio. Dentro del mercado de seguros, la asimetría informativa se ve reflejada principalmente en la complejidad y especialización de los contratos que limitarían la posibilidad del consumidor para poder tomar una decisión de consumo debidamente informada. Esta complejidad, como apuntó Howard Davies (Davies 1998), podría verse agravada incluso por la problemática que representa el largo plazo de las relaciones de consumo, donde el retorno esperado podría no presentarse sino muchos años después de la contratación de un servicio determinado.

Por otro lado, para la teoría económica, la asimetría informativa es percibida como una falla del mercado. Sin embargo, es importante poner ello en contexto para entender con mayor claridad qué es lo que el Estado desea proteger o qué falla del mercado tendría interés en solucionar.

En consecuencia, la asimetría informativa no debe ser entendida como una diferencia de conocimiento (entre el proveedor y el consumidor) que va necesariamente en desmedro del consumidor, pues ello no tendría en cuenta que es precisamente la existencia de ese conocimiento asimétrico lo que permite la existencia de proveedores y consumidores en primer lugar (como parte del fenómeno económico de división del trabajo). Para entender esto último, pongamos como ejemplo cualquier bien que se comercie en el mercado (un perfume); la compañía que elabora el perfume tiene más información y conocimiento sobre la elaboración de este producto (insumos, tecnología, contenido, esquema de negocio, entre otros) y es precisamente ello lo que permite que su elaboración sea menos costosa para la compañía que para el consumidor, y que la transacción mediante la cual el consumidor adquiere el bien del proveedor sea económicamente más eficiente que si el propio consumidor elaborara su propio perfume sin la intervención del proveedor.

Entonces, la existencia de una asimetría informativa no constituye per se y en sí misma, una falla del mercado; sino que es un elemento fundamental para la existencia del mismo y para la celebración de transacciones comerciales eficientes.

Conviene tener presente, en esa misma línea, que la asimetría informativa no es entonces una situación indeseable per se que debe ser evitada a toda costa. En este sentido nos parece acertado lo comentado por Alfredo Bullard quien señala que:

"La asimetría informativa es parte del fenómeno económico de división del trabajo, esto es, que algunos en la sociedad nos dedicamos a ciertas actividades (usualmente las que conocemos mejor) y otros a otras. El cliente de un abogado no aspira a tener información simétrica con él. Si fuera así iría a la universidad a estudiar Derecho. Justamente lo contrata para no tener que estudiar Derecho. El cliente posiblemente sepa más que el abogado respecto de sus 


\section{Seguros de grandes riesgos y protección desproporcionada de los asegurados en la Ley del contrato de seguros Great risk insurances and disproportionate protection of insured persons in insurance contract Law}

\begin{abstract}
propios negocios. Es justamente la diferencia de información lo que hace eficiente el intercambio y permite que cada quien en la sociedad se especialice y aumente la productividad de una manera asombrosa, algo que no ocurriría si uno mismo tuviera que producir todos los bienes y servicios que necesita, lo que significaría saber de todo. (...) Lo que debe evitarse es que, en la contratación, esa asimetría genere transacciones ineficientes, porque son tomadas sin una adecuada evaluación de costos y beneficios. Por ello, el objetivo no es la simetría informativa, sino la información adecuada, entendiendo por 'adecuada' aquella cuya producción para el consumidor cuesta menos que los beneficios que genera" (Bullard 2010) (la cursiva es agregada).
\end{abstract}

En conclusión, sería un sinsentido que el Estado pretenda erradicar esta característica del mercado, buscando la simetría informativa como el nirvana del desarrollo y un objetivo en sí mismo. En efecto, el objeto de la regulación debe ser entendido únicamente como la reducción de la asimetría informativa entre proveedores y consumidores, tal como ha sido expresado por el Artículo II del Título Preliminar del Código de Protección y Defensa del Consumidor antes citado, con la finalidad de permitir que el consumidor tome una decisión de consumo informada, evaluando los posibles costos y beneficios que se pueden derivar de una transacción; pero de ninguna forma, la norma debe ser entendida como una obligación de divulgación completa de todo tipo de información al mercado o de supresión total de la asimetría informativa, en tanto esta interpretación y el costo regulatorio que ello implique no serían razonables, tomando en cuenta las características del mercado descritas en cuanto al manejo de la información y la necesidad de contar con división del trabajo en un sistema que se sustenta en una economía de mercado.

En cualquier caso, el rol protector del Estado en materia de consumo se justifica esencialmente (o por lo menos en una sociedad de respeto por las libertades individuales) en la necesidad de que los consumidores puedan tomar decisiones informadas sobre la contratación de bienes y servicios; en tal sentido, y como señala Gustavo Rodríguez García, "la racionalidad económica de la normativa de protección al consumidor está enfocada a la previsión y no a la intervención directa del Estado" (Rodríguez 2008).

La asimetría informativa, entonces, dejará de ser relevante en una relación de consumo cuando el usuario del servicio tenga capacidad de negociación y recursos suficientes (asesoría especializada, experiencia previa en negociaciones de este tipo y en los riesgos que afectan la industria en las que el tomador del seguro opera, usualmente con la posibilidad de contar con profesionales con experiencia y sofisticación en este tipo de negociaciones, entre otros) para poder tomar una decisión informada. Por lo tanto, en un escenario en el que la asimetría informativa es irrelevante porque el consumidor cuenta con la información suficiente para tomar una decisión evaluando potenciales costos y beneficios, no se justifica en modo alguno la intervención estatal.

\section{Interpretación del artículo III de las Disposiciones Generales de la Ley del Contrato de Seguro}

Cierta doctrina nacional ha sostenido que los seguros de grandes riesgos ya se encuentran excluidos del ámbito de aplicación de la vigente Ley del Contrato de Seguro en vista que el Artículo III de sus Disposiciones Generales establece que "el contrato de seguro se celebra por adhesión, excepto en las cláusulas que se hayan negociado entre las partes y que difieran sustancialmente con las pre redactadas".

De esta forma, se sostiene que el contrato de seguro sólo sería protegido por la Ley (y otras normas reglamentarias y regulatorias que se emitan) en caso se trate de un contrato celebrado por adhesión y que, si no se ha celebrado por adhesión, la empresa de seguros tendría que acreditar, ante el Poder Judicial o ante el INDECOPI (o, de ser el caso, ante los árbitros que conozcan potenciales controversias en materia de seguros), que el tomador negoció el contrato de seguros respectivo de igual a igual, acreditando la existencia y alcances de tal negociación 


\section{Rafael Corzo de la Colina y José Villafuerte Mendoza}

con los cambios introducidos por el tomador en la póliza de seguros respectiva.

Consideramos que este argumento es, por lo menos, discutible por las siguientes razones:

a) La inexistencia de un contrato celebrado por adhesión se determina por la capacidad de negociación del tomador (en el sentido potencial del término) y no por el efectivo ejercicio de esa capacidad que se materialice a través de la inclusión de ciertas modificaciones en dicho contrato, pues en ese caso, caeríamos en el absurdo de que un mismo tomador sea protegido bajo los alcances de una póliza en la que no realizó modificaciones (por decisión propia) y no sea protegido en otra póliza en la que efectuó modificaciones haciendo uso de su capacidad de negociación, todo ello debido al elevado estándar de prueba que se propone (esto es, demostrar las modificaciones introducidas por el asegurado).

Si un tomador ha tenido cierta capacidad de negociación, independientemente de que la haya ejercido en la práctica o no, no debería determinar por esta situación que se debería encontrar protegido (o no) por la Ley del Contrato de Seguro, pues pueden existir muchas razones por las cuales pueda haber decidido no realizar o proponer modificaciones en el contrato de seguro materia de negociación (esto es, la póliza era estándar en el mercado, las condiciones de la póliza le parecían razonables, entre otras razones o circunstancias que podrían presentarse) y eso no afecta la capacidad de negociación del tomador y menos la poca relevancia de la brecha de asimetría informativa que podría existir entre el tomador y la empresa de seguros.

b) Por otro lado, una interpretación como la que propone cierta doctrina nacional podría generar incentivos perversos en ciertos tomadores, quienes, en una negociación, podrían adoptar la postura de no efectuar modificaciones en la póliza bajo la premisa de que la Ley del Contrato de Seguro los va a proteger en aquello que pudieron negociar, desnaturalizando el rol protector del Estado en este tipo de supuestos.

c) Asimismo, una determinación, mediante control difuso, de la aplicabilidad de la Ley del Contrato de Seguro, considerando la poca predictibilidad del Poder Judicial y la especialidad existente en materia de derecho de seguros y, eventualmente, las distintas industrias y tipo de operaciones que son materia de cobertura, encarecerá más la regulación a la que se encuentran sometidas las empresas de seguros, pues en cualquier supuesto las partes vinculadas a una operación de seguros tendrían que adoptar una posición conservadora sobre la interpretación de la norma (haciendo inaplicable en la práctica la norma del artículo III de las Disposiciones Generales de la Ley del Contrato de Seguros), además del hecho que no estamos de acuerdo en que la sola existencia de esta facultad discrecional de los Jueces y Tribunales pueda servir para sustentar una posición jurídica.

d) No existe argumento para que el rol protector del Estado tenga un estándar distinto de aplicación entre la Ley del Contrato de Seguro y el Código de Protección al Consumidor. Existen muchos productos o servicios cuya trascendencia social (si se desea utilizar el término) es comparable con los seguros, razón que, desde nuestro punto de vista, no justificaría que el estándar de protección difiera entre tales supuestos.

Asimismo, en el caso de la función tuitiva y de protección por parte del Estado en la constitución del Fondo de Seguro de Depósito, se puede apreciar que el mismo sólo se extiende a favor de las personas naturales $y$ de las personas jurídicas sin fines de lucro, conforme a lo establecido por el artículo 152 de la Ley General de los Sistemas Financieros y de Seguros y, en ningún caso, para el supuesto de los depósitos constituidos por personas jurídicas, con excepción de los depósitos a la vista (esto es, solamente los saldos acreedores depositados en cuentas corrientes, pero no a otras modalidades de 
Seguros de grandes riesgos y protección desproporcionada de los asegurados en la Ley del contrato de seguros Great risk insurances and disproportionate protection of insured persons in insurance contract Law

imposiciones y de ahorro). Esta regulación demuestra también la existencia del estándar diferenciado con el que el Estado estaría ejerciendo su rol protector.

\section{Consecuencias de la protección desproporcionada de los asegurados en seguros de grandes riesgos}

Como hemos adelantado en puntos anteriores, tomando en cuenta que la razón de ser del rol protector del Estado radica en la asimetría informativa de una relación de consumo, y que esta deviene en irrelevante cuando se trata de situaciones en las que el consumidor o, para nuestros efectos, el tomador del seguro, cuenta con capacidad de negociación y recursos suficientes para poder tomar una decisión de consumo informada, no existe racionalidad económica para aplicar cualquier regulación tuitiva a una situación en la que la asimetría informativa es irrelevante, como podría ser el caso de los seguros de grandes riesgos a los que hemos hecho referencia en las primeras secciones del presente artículo.

Por otro lado, una sobrerregulación o protección a los tomadores de seguros y asegurados en los seguros de grandes riesgos no solamente carece de racionalidad económica, por los argumentos expresados, sino que también resulta contraproducente a los fines de la regulación sobre protección al consumidor.

Al respecto, desde nuestro punto de vista, la aplicación de normas tuitivas (Ley del Contrato de Seguro o normas generales sobre protección al consumidor) a los tomadores de seguros de grandes riesgos, ocasiona, principalmente, un encarecimiento innecesario de la regulación para las aseguradoras, situación que se va a ver reflejada directamente en el costo de las primas de seguro que son ofrecidas en el mercado.

Asimismo, esta sobrerregulación podría generar incentivos perversos y ventajas indebidas a ciertos tomadores de seguros, quienes sí cuentan con recursos y capacidad de negociación frente a una empresa de seguros, desnaturalizando de este modo el rol protector del Estado y generando un clima de inseguridad jurídica respecto del ámbito de aplicación de la Ley del Contrato de Seguro (el mismo que se determinaría a través de un control difuso, como ha propuesto cierto sector de la doctrina).
Estas situaciones se traducen en un mayor riesgo de la cartera que es administrada por las aseguradoras, y, consecuentemente, incrementan el riesgo protegido por los contratos de reaseguro. En ese orden de ideas, el mayor riesgo que asuman las empresas de reaseguros generará, a su vez y también, un encarecimiento en sus primas; sobrecosto que, a la larga, será trasladado, en mayor o menor medida, al consumidor final (tomador del seguro), afectando al mismo y, por otro lado, reduciendo la competitividad, solvencia y confiabilidad que debería tener el Sistema de Seguros Peruano, tal como lo dispone el artículo 2 de la Ley General de los Sistemas Financiero y de Seguros vigente en el Perú.

\section{Propuesta de tratamiento normativo de los seguros de grandes riesgos}

Entendiendo que el tratamiento especial de los seguros de grandes riesgos se encuentra orientado a evitar una sobrerregulación y a proponer una solución eficiente donde un régimen de protección excesivo de parte del Estado en estos casos podría resultar contraproducente, consideramos necesaria la exclusión de los grandes riesgos del ámbito de aplicación de la Ley del Contrato de Seguro, así como de cualquier otra norma protectora de los intereses del tomador y que pretenda sustituir la voluntad de las partes.

Consideramos pertinente, en consecuencia, equiparar la aplicación de la Ley del Contrato de Seguro peruana a estándares internacionales, excluyendo de su aplicación a seguros de grandes riesgos, pudiendo adoptar para ello alguna de las siguientes alternativas:

a) Establecer criterios objetivos de exclusión de determinados riesgos, entre los que se podrían tener en consideración, por ejemplo, el monto de prima pactada, el 


\section{Rafael Corzo de la Colina y José Villafuerte Mendoza}

valor total de los bienes asegurados, las condiciones económicas y financieras del tomador del seguro, la naturaleza del riesgo o la actividad económica del tomador, entre otros criterios; o,

b) Equiparar el tratamiento legislativo, el ámbito de aplicación y los alcances de la Ley del Contrato de Seguro peruana, respecto de los seguros que cubren los denominados grandes riesgos, al ámbito de aplicación y los alcances que han sido establecidos por el legislador para el caso del Código de Protección al Consumidor. Tomando en cuenta que ambas normas desarrollan el rol protector del Estado en materia de consumo, resulta inconsistente que ambas disposiciones legales tengan ámbitos de aplicación y alcances que sean tan distintos.

\section{Referencias bibliográficas}

Espinoza Espinoza, Juan. 2004. Ley de Protección al Consumidor. Lima: Editorial Rodhas.

Bullard, Alfredo. 2010. ¿Es el Consumidor un Idiota? El Falso Dilema entre el Consumidor Razonable y el Consumidor Ordinario. Ensayos sobre Protección al Consumidor en el Perú. Revista de la Competencia y la Propiedad Intelectual 10 (Marzo): 5-58.

Davies, Howard. 1998. Why Regulate? Conferencia presentada en "Henry Thornton Lecture", en City University Business School, Londres. http://www.fsa.gov.uk/Pages/ Library/Communication/Speeches/1998/sp19.shtml

Rodríguez García, Gustavo. 2008. ¿Asimetría informativa o desigualdad en el mercado?: apuntes sobre el verdadero rol de la protección al consumidor. Foro Jurídico 8: 113-9. 